\title{
MEMS-based Measurement of Rheological Fluid Properties
}

\author{
I. Dufour ${ }^{1}$, E. Lemaire ${ }^{1}$, B. Caillard ${ }^{1}$ \\ ${ }^{1}$ Univ. Bordeaux, IMS, UMR 5218, F-33400 Talence, France, \\ isabelle.dufour@ims-bordeaux.fr
}

\begin{abstract}
:
Silicon microcantilevers can be used to measure the rheological properties of complex fluids. In the method presented in this paper, the measurement of the microcantilever's dynamic spectrum is used to extract the hydrodynamic force exerted by the surrounding fluid on the microcantilever and consequently the rheological fluid properties. The originality of the proposed method relies in the fact that not only may the viscosity of the fluid be measured but also the fluid's viscoelasticity, i.e., both viscous and elastic properties, which are key parameters in the case of complex fluids. In this method the use of analytical equations allows the fluid's complex shear modulus to be extracted and expressed as a function of the frequency. Viscoelastic spectral characteristics of fluids can be determined in analogy to rheograms recorded with standard laboratory equipment, and extend the frequencies of investigation to the range $1-100 \mathrm{kHz}$.
\end{abstract}

Key words: Microcantilever, rheology, viscoelasticity, hydrodynamic force, MicroElectroMechanical Systems (MEMS), complex fluids

\section{Context and objectives}

The aim of the presented work is to investigate new rheological microsensors both for improving the fundamental comprehension of the behavior of soft matter and to address issues concerning the processing, quality control and formulation of industrial products.

Concerning the fundamental approach, classical rheological measurement systems using oscillatory vibrations are limited to very low frequency (under few hundreds Hertz). The properties of many complex fluids are frequency dependent and their characterization over a larger frequency range is interesting to point out some physical phenomena. For example, if an entangled polymer network is excited with characteristic times longer than the time constants associated with untangling the polymer chains, the network will untangle and become viscous as a simple liquid. On the contrary, if the excitation is made on shorter times, the complex fluid will stay an entangled network and it will exhibit a significant viscoelastic behavior. These phenomena have been widely studied in literature. Our MEMSbased devices allow enlarging the frequency dependent study and particularly for the range between $100 \mathrm{~Hz}$ and $100 \mathrm{kHz}$ (not covered by the classical rheometers and the quartz crystal microsensors).
Concerning the impact on the industry, we have to remember that rheology is defined as the science of deformation and flow of matter. Additives, also called 'rheological modifiers' are used to give specific characteristics to the flow of a product and it is the formulation chemist who is in charge of combining many ingredients to form a stable composition with specific rheological properties. Rheological modifiers span the full spectrum of applications ranging from food, personal care and pharmaceutical industry to oil field and drilling chemicals, inks, polymers, paints, household products, adhesives, etc. In all these various industries, the common objective is to achieve the desired flow consistency of the end product. Alternatively, rheological properties monitoring may serve as an indirect means to detect spurious changes in a process involving liquids, which, e.g., leads to applications in so-called condition monitoring systems. In summary, the rheology of fluid-based products has a high impact on their characterization before industrialization. Rheological instruments and devices represent a huge and rapidly growing market, which has a significant impact on quality standards and safety in case of critical products and processes.

Rheological instruments play a central role in the characterization of liquids both for fundamental research and for industrial process control applications. As rheological measurements require the generation of 
accurate shear movements within liquids, traditional instruments rely on the technology of precision engineering and mechanics. Novel fabrication technologies, partly triggered by the rapidly evolving field of microsystems technology, have recently led to the investigation of novel miniaturized devices that can be used for rheometric sensing and measurement utilizing vibrating structures. These technologies yield smaller devices which commonly also require less maintenance than the established laboratory devices and are thus suitable for integration in condition monitoring systems and in situ industrial process control. Moreover, as this will be outlined in the following sections they also allow exploring the rheological properties over a wider frequency range than that covered by classical rheometers.

\section{MEMS-based rheological sensors}

The classical way to measure the rheological properties of fluids is to use rheometers (coneplate or Couette geometries [1]). But there are some drawbacks when using such instruments: the amount of fluid required to make the measurement is quite large (a few milliliters) and the measurement is limited to low frequencies (less than $100 \mathrm{~Hz}$ ).

To overcome the frequency range limitation some alternative methods have been developed at the micro-scale, which can be referred to as 'microrheology'. A first alternative consists of monitoring the movement of monodisperse beads (microspheres) immersed in the fluid [2-8]. The beads motion can be active (optical or magnetic tweezers) or passive (Brownian motion) and the acquisition of the motion is performed optically. The major drawbacks of such devices are the long image and signal processing to extract the fluid rheological properties and a possible interaction between the fluid and the beads. The analysis of the experimental data may also be troublesome and complex situations are encountered when the size of the sensing particles becomes smaller than the mesoscopic scale of the sample (mesh size of a gel for example). In this case, the beads do not feel the gel structure and diffuse as if they were immersed in the solvent.

In order to cover higher frequencies (from $1 \mathrm{MHz}$ to a few hundreds of $\mathrm{MHz}$ ), acoustic shear-polarized devices, such as QCM or Love wave devices, have been used as a second alternative [9-14]. These high-frequency devices are very compact but they also show adverse properties: (i) the penetration depth of the shear wave in the liquid is very low so that only a very thin film viscoelasticity is measured; (ii) the displacement amplitudes are small so that non-linear effects requiring a certain minimum stimulation in terms of spatial displacements to occur may not be activated and thus are not being sensed. The first item also leads to issues when samples having microstructures with characteristic lengths larger than the penetration depth are investigated, such as may be the case for suspensions and emulsions [15].

At IMS Laboratory, we are aiming at investigating and devising alternative methods based on the use of vibrating micro-electromechanical systems (MEMS). Some other teams have also used vibrating microstructures to measure viscosity at various frequencies [1619]. They use the fact that both the resonant frequency and the quality factor depend on the fluid density and viscosity. In this approach the estimation is usually made after calibration steps. The major drawbacks of such approaches stem from the fact that when using only one microstructure viscosity is determined at only one frequency and the elasticity of the fluid is neither taken into account nor estimated.

The previous major contributions of IMS Laboratory in rheological MEMS-based microsensors concern three different methods:

- Unlike other teams, in order to extract both the fluid viscosity and the mass density, the approaches devised by IMS Laboratory do not require any calibration step except the measurement of the resonant frequency in air. Using the analytical equations obtained from the solution of the differential equation of the vibration of a microcantilever in a fluid and an approximation of the hydrodynamic function we express the mass density and viscosity of the fluid from both the eigenfrequency and damping ratio measurement [20]. The limitation of this method is that fluid properties are determined only at one frequency per device.

- To avoid this drawback, another method has been developed that consists in determining the hydrodynamic force components (viscous and inertial) at each frequency of the spectrum measurement. Using analytical equations the mass density and fluid viscosity can in this case be estimated at each frequency [21-22]. The limitation of this method is that analytical equations only work for pure viscous fluids considering the elastic contribution negligible.

- Knowing the mass density of the fluid under test, another method, based on the same 
principle has been developed to estimate both elastic and viscous components of the shear modulus as a function of frequency [23].

The principle and the results of the last method will be presented in this paper since it is quite easy to determine the mass density of a fluid by other means.

\section{Basic principle of MEMS rheological sensors}

When a vibrating cantilever is immersed in a fluid, the fluid exerts a hydrodynamic force on the cantilever due to both the pressure force on the surfaces perpendicular to the cantilever displacement and the shear force on the surfaces parallel to the cantilever displacement. The total hydrodynamic force is composed of two terms: one inertial term proportional to microcantilever acceleration and one viscous term proportional to microcantilever velocity. The microcantilever deflection in the Fourierspace is governed by the Euler-Bernoulli equation:

$$
\begin{aligned}
& E I \frac{\partial^{4} w(\omega, x)}{\partial x^{4}}-m_{L} \omega^{2} w(\omega, x)= \\
& F(\omega, x)+F_{\text {fluid }}(\omega, x)
\end{aligned}
$$

where:

- $x$ is the coordinate along the cantilever length, $y$ along the cantilever width and $z$ along the cantilever thickness,

- $w(\omega, x)$ is the deflection in the $z$ direction at coordinate $x$ and at radial frequency $\omega$,

- $E$ is the Young's modulus of the cantilever material,

- $I$ is the second moment of area at coordinate $x$ with respect to the $y$ axis,

- $F(\omega, x)$ is the actuation force per unit length at coordinate $x$. In the case of a concentrated force at the free-end: $F(\omega, x)=F_{0}(\omega) \delta(x-L), \delta$ being the Dirac function,

- $F_{\text {fluid }}(\omega, x)$ is the force per unit length exerted by the fluid on the cantilever at coordinate $x$,

- $m_{L}$ is the mass per unit length of the microcantilever.

The hydrodynamic force can be expressed:

$$
F_{\text {fluid }}(\omega, x)=\left(-g_{1}-j \omega g_{2}\right) j \omega w(\omega, x)
$$

with the terms $g_{1}$ and $g_{2}$ representing the viscous and inertial effects respectively.

According to [24] the following simple expressions may be used to quantify the hydrodynamic force: $g_{1}=\frac{\pi}{4} \rho b^{2} \omega\left(b_{1} \frac{\delta}{b}+b_{2}\left(\frac{\delta}{b}\right)^{2}\right)$

and

$g_{2}=\frac{\pi}{4} \rho b^{2}\left(a_{1}+a_{2} \frac{\delta}{b}\right)$

with

- $\rho$ the mass density of the microcantilever,

- $b$ the width of the microcantilever,

$-\delta=\sqrt{\frac{2 \eta}{\rho_{f} \omega}}$ the fluid layer thickness over which the imposed cantilever velocity decays by a factor of $e=2.72$; with $\eta$ the fluid viscosity and $\rho_{f}$ the fluid mass density,

- for physical reasons, the values parameters are taken as follow $a_{1}=1.000, a_{2}=b_{1}=3.8007$ and $b_{2}=3.8437$.

The solution of Eq. 1 depends on the hydrodynamic force which, according to Eqs. 24 , depends on the fluid density and viscosity.

Eqs. 1-4 correspond to the case of a Newtonian fluid (that means a fluid with no elastic properties). In the case of complex fluids, Eqs. 3-4 should be modified since the rheological properties are now characterized either by a complex viscosity $\eta^{*}$ or a complex shear modulus $G^{*}$ defined by:

$$
\begin{aligned}
& \eta^{*}=\frac{\tau}{\dot{\gamma}}=\eta^{\prime}-j \eta^{\prime \prime} \\
& G^{*}=\frac{\tau}{\gamma}=G^{\prime}+j G^{\prime \prime}=j \omega \eta^{*}=j \omega\left(\eta^{\prime}-j \eta^{\prime \prime}\right)
\end{aligned}
$$

with $\tau$ being the shear stress, $\gamma$ the shear strain, $\dot{\gamma}$ the shear rate and $\eta^{\prime}, \eta^{\prime \prime}, G^{\prime}$ and $G$ " being, respectively, the real and imaginary parts of the viscosity, the elastic shear modulus, and the viscous shear modulus. The 'standard' unit of viscosity is Pa.s, but usually the centiPoise (cP) is used, which corresponds to 0.001 Pa.s (the viscosity of water). The unit of the shear modulus is $\mathrm{Pa}$. This complex notation for both the viscosity and the shear modulus reveals the possible phase shift between shear stress and shear strain or shear rate. A perfect liquid has a zero elastic modulus $G^{\prime}$ whereas a perfect solid has a zero viscous modulus $G$ ". 


\section{Silicon MEMS and example of measurements}

In order to measure the spectrum of microcantilevers in different fluids, chips with rectangular silicon-based microcantilevers were provided by LAAS-CNRS at Toulouse, France. Series of microcantilevers were used for measurements, namely $L L, L H$ and $A$, having the different geometries specified in Tab. 1 (due to the use of SOI wafers the silicon thicknesses of all microcantilevers are the same). The microcantilevers have been designed in order to be actuated electromagnetically by the Lorentz force. For this purpose, a conducting path has been deposited on their top surface (Fig. 1). The conducting path is made of a titanium layer of $100 \mathrm{~nm}$ thickness covered by a gold layer of $700 \mathrm{~nm}$ thickness. The width of the conducting path is $10 \mu \mathrm{m}$ and is placed at $10 \mu \mathrm{m}$ from the edge of the cantilever. The electromagnetic actuation is obtained by the use of either one (or two) magnet(s) generating a continuous magnetic field and by an alternative voltage generating an alternative electrical current in the conducting path. Then, the Lorentz force causes the microcantilever deflection at the same frequency as the actuation voltage. In order to be able to make measurements in electrically conductive fluids, a layer of silicon oxide is spread on the top surface of the cantilever, above the conducting path: the thickness of this insulating layer is $600 \mathrm{~nm}$.

Tab. 1: Geometry of the three microcantilevers

\begin{tabular}{|l|l|l|l|}
\hline Geometry & LL & LH & A \\
\hline Length $(\mu \mathrm{m})$ & 2810 & 1440 & 500 \\
\hline Width $(\mu \mathrm{m})$ & 100 & 285 & 100 \\
\hline Thickness $(\mu \mathrm{m})$ & 20 & 20 & 20 \\
\hline
\end{tabular}

Fig. 1. Schematic representation of the microcantilever with a conducting path allowing the electromagnetic actuation using a magnet and an alternative voltage.
Vibration measurements have been carried out using an optical vibrometer allowing out-ofplane measurements using Doppler interferometry (MSA 500 Polytec). Examples of spectra measurements are presented in Fig. 2.
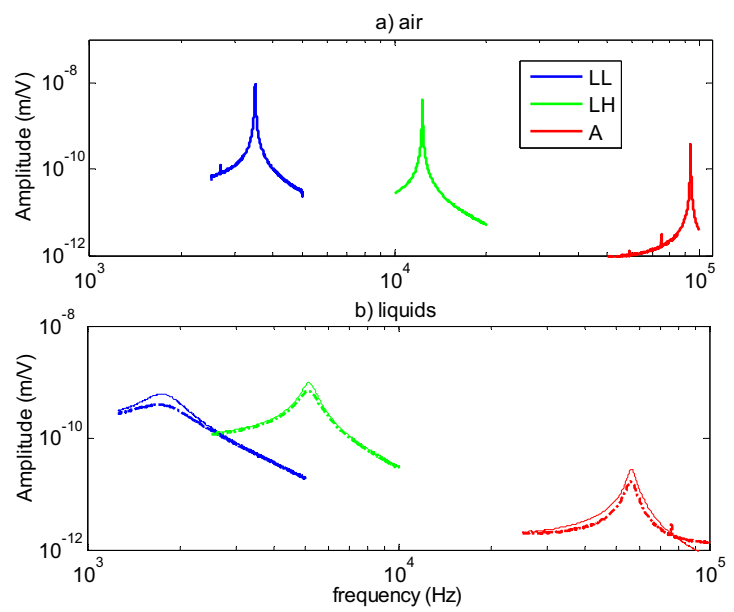

Fig. 2. Measurements of cantilevers LL (blue), LH (green) and $A$ (red). a) Resonant peaks into air. b) Damped resonance into water (lines) and into polyacrylamide 1000 ppm (dotted).

As can be seen in Fig. 2, the microcantilever spectrum depends on the properties of the surrounding fluid. Moreover, the frequency range for which the spectrum is modified depends on the geometry of the cantilever. Therefore, each microcantilever can be used for a specific frequency range: the $L L$ cantilever is for the lowest frequency range, the $\mathrm{LH}$ cantilever for a low-mid frequency range and the $A$ cantilever for the higher frequency range.

\section{Estimation of the rheological fluid properties}

Using the amplitude deflection spectrum measurements, the phase deflection spectrum measurements and analytical equations based on the simplification of the solution of Eq. 1, the values of the terms $g_{1}$ and $g_{2}$ at each frequency of measurement are calculated. Then, based on Eqs. 3-4 adapted with a complex viscosity expression $\eta^{*}$, the elastic and viscous shear moduli can be expressed:

$G "=\frac{g_{1}}{D}-\frac{B}{D \sqrt{2 D}} \sqrt{\sqrt{\left(\frac{B^{2}}{D}+2\left(g_{2} \omega-C\right)\right)^{2}+4 g_{1}^{2}}}$

$G^{\prime}=\frac{1}{D}\left(g_{2} \omega-C-\frac{B^{2} G^{\prime \prime}}{g_{1}-D G^{\prime \prime}}\right)$

With: 


$$
B=\frac{\pi b_{1}}{2 \sqrt{2}} b \sqrt{\rho_{f}} \quad C=\frac{\pi}{4} \rho_{f} b^{2} \omega \quad D=\frac{\pi b_{2}}{2 \omega}
$$

The calculation of $G$ ' and $G$ " for any radial frequency $\omega$ can then be performed provided that $g_{1}$ and $g_{2}$ can be estimated with enough accuracy from the spectrum measurements.

The rheogram presented in Fig. 3 illustrates the Newtonian behavior of water obtained with the three cantilevers spectrum measurements: more than one decade separates both moduli so $G$ ' can be considered negligible compared to G".

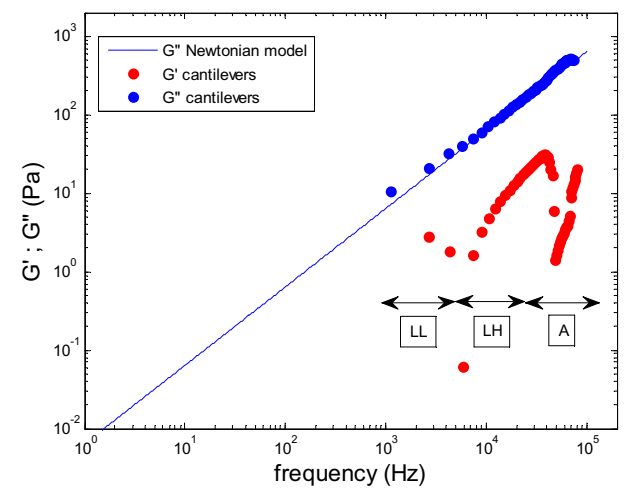

Fig. 3. Rheogram of water measured with three microcantilevers ( $L L, L H$ and $A$ ) and covering the frequency range $10^{3}-10^{5} \mathrm{~Hz}$ using Eqs. 7-9.

Complex fluids have also been characterized. An example of characterization of CTAB with microcantilevers at $19^{\circ} \mathrm{C}$ is given in Fig. 4 with a comparison with measurements made with the same concentration of polymer by GalvanMiyoshi et al. at $22^{\circ} \mathrm{C}$ [25] and cone-plate measurements made at $19^{\circ} \mathrm{C}$.

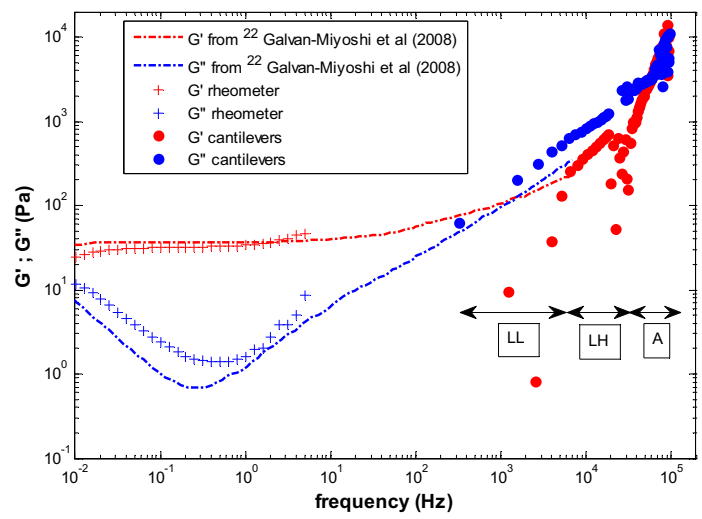

Fig. 4. Rheogram of CTAB measured with a classical cone-plate rheometer and three cantilever geometries ( $L L, L H$ and $A$ ) and covering the frequency range $10^{3}-10^{5} \mathrm{~Hz}$ using Eqs. 7-9. Comparison with literature DWS measurements [25].
In this case, unlike Newtonian fluids, $G^{\prime}$ is not negligible anymore. Except for $G$ ' measurements obtained with $L L$ cantilevers $\left(G_{L L}^{\prime}\right)$, all sets of measurements of $G^{\prime}$ and $G$ " clearly follow the same trends until $100 \mathrm{kHz}$, given the temperature difference.

\section{Conclusion}

Microcantilevers can probe the $[1-100 \mathrm{kHz}]$ frequency viscoelastic properties of complex fluids, such as gels and polymers that are of high interests both for fundamental research and for industrial purposes. This is possible thanks to (i) MEMS manufacturing technology (ii) theoretical developments (iii) accurate measurements. For in-situ and real time monitoring of all kinds of fluids (even opaque fluids), the third item still must be developed so that electrical measurements can replace optical measurements.

\section{Acknowledgements}

The authors would like to thank Cédric Ayéla for the cantilevers mask design, Liviu Nicu, Fabrice Mathieu and Daysuke Saya for the fabrication of the silicon cantilever. This work was partially supported by the CPER Pôle 4N Nanosciences en Aquitaine (GP-206-action 216/1) with the contribution of the Conseil Régional d'Aquitaine, the FEDER and the Ministry of Education and Research, by the French National Agency (MicRheo project $n^{\circ}$ ANR-08NANO-004) and by the Conseil Régional d'Aquitaine ( $\left.n^{\circ} 20091102001\right)$.

\section{References}

[1] F.A. Morrison, Understanding rheology, Oxford University Press, (2001). ISBN: 978-0-19514166-5

[2] V. Breedveld, D.J. Pine, Microrheology as a tool for high-throughput screening, Journal of Materials Science, 38, 4461-4470 (2003). doi:10.1023/A:1027321232318.

[3] F.C. MacKintosh, C.F. Schmidt, Microrheology, Current Opinion in Colloid \& Interface Science, 4, 300-307 (1999). doi:10.1016/S13590294(99)90010-9.

[4] T.A. Waigh, Microrheology of complex fluids, Reports on Progress in Physics, 68, 685-742 (2005). doi:10.1088/0034-4885/68/3/R04.

[5] M.L. Gardel, M.T. Valentine, D.A. Weitz, Microrheology, in Microscale Diagnostic Techniques. K.S. Breuer, Springer Verlag Berlin Heidelberg (2005).ISBN: 3-540-23099-8.

[6] N. Willenbacher, C. Oelschlaeger, Dynamics and structure of complex fluids from high frequency mechanical and optical rheometry, Current Opinion in Colloid \& Interface Science, 12, 43-49 (2007). doi:10.1016/j.cocis.2007.03.004. 
[7] F. Ortega, H. Ritacco, R.G. Rubio, Interfacial microrheology: Particle tracking and related techniques, Current Opinion in Colloid \& Interface Science, 15, 237-245 (2010). doi: 10.1016/j.cocis.2010.03.001.

[8] P. Cicuta, A.M. Donald, Microrheology: a review of the method and applications, Soft Matter, 3, 1449-1455 (2007). doi: 10.1039/b706004c.

[9] B. Jakoby, M. J. Vellekoop, Viscosity sensing using a Love wave device, Sensors and Actuators A, 68, 275-281 (1998). doi:10.1016/S0924-4247(98)00017-X.

[10] B. Jakoby, M. J. Vellekoop, Properties of Love waves: applications in sensors, Smart Materials and Structures, 6, 668-679 (1997). doi:10.1088/0964-1726/6/6/003.

[11] B. Jakoby, M. Scherer, M. Buskies, H. Eisenschmid, An automotive engine oil viscosity sensor, IEEE Sensors Journal, 3, 562-568 (2003). doi:10.1109/JSEN.2003.817164.

[12] S.J. Martin, G.C. Frye, K.O. Wessendorf, Sensing liquid properties with thickness-shear mode resonators, Sensors and actuators $A, 44,209$ 219 (1994). doi:10.1016/0924-4247(94)00806-X.

[13] S.J. Martin, V.E. Granstaff, G.C. Frye, Characterization of a Quartz Crystal Microbalance with simultaneous mass and liquid loading, Analytical Chemistry, 63, 2272-2281 (1991). doi:10.1021/ac00020a015.

[14] D.C. Ash, M.J. Joyce, C. Barnes, C.J. Booth, A.C. Jefferies, Viscosity measurement of industrial oils using the droplet quartz crystal microbalance, Measurement Science and Technology, 14, 1955-1962 (2003). doi:10.1088/0957-0233/14/11/013.

[15] B. Jakoby, R. Beigelbeck, F. Keplinger, F. Lucklum, A. Niedermayer, E. Reichel, C. Riesch, T. Voglhuber-Brunnmaier, B. Weiss, Miniaturized sensors for the viscosity and density of liquids performance and issues, IEEE Transactions on Ultrasonics, Ferroelectrics and Frequency Control, 57, 111-120 (2010). doi:10.1109/TUFFC.2010.1386.

[16] C. Bergaud, L. Nicu, Viscosity measurements based on experimental investigations of composite cantilever beam eigenfrequencies in viscous media, Review of Scientific Instruments, 71, 2487-2491 (2000). doi:10.1063/1.1150640.

[17] S. Boskovic, J. W. M. Chon, P. Mulvaney and J.E. Sader, Rheological measurements using microcantilevers, Journal of Rheology, 46, 891899 (2002). doi:10.1122/1.1475978.

[18] M. Hennemeyer, S. Burghart, R. W. Stark, Cantilever micro-rheometer for the characterization of sugar solutions, Sensors, 8, 10-22 (2008). doi:10.3390/s8010010.

[19] A. Agoston, F. Keplinger, B. Jakoby, Evaluation of a vibrating micromachined cantilever sensor for measuring the viscosity of complex organic liquids, Sensors and Actuators A, 123-124, 82-86 (2005). doi:10.1016/j.sna.2005.02.020.

[20] M. Youssry, N. Belmiloud, B. Caillard, C. Ayela, C. Pellet, I. Dufour, A straightforward determination of fluid viscosity and density using microcantilevers: from experimental data to analytical expressions, Sensors and Actuators A, 172, 40-46 (2011). doi:10.1016/j.sna.2011.02.025

[21] N. Belmiloud, I. Dufour, A. Colin, L. Nicu, Rheological behaviour probed by vibrating microcantilevers, Applied Physics Letters, 92, 041907 (2008). doi:10.1063/1.2837181.

[22] M. Youssry, B. Caillard, C. Ayela, C. Pellet, I. Dufour, Microrheology of newtonian fluids using microcantilever, The Second IASTED International Conference on Nanotechnology and Applications, NANA 2010, 540-546 (2010). doi:10.2316/P2010.707-018

[23] M. Youssry, E. Lemaire, B. Caillard, A. Colin, I. Dufour, On-chip characterization of the viscoelasticity of complex fluids using microcantilevers, Measurement Science and Technology, 23, 125306 (2012). doi:10.1088/0957-0233/23/12/125306.

[24] A. Maali, C. Hurth, R. Boisgard, C. Jai, T. CohenBouhacina, J-P. Aimé, Hydrodynamics of oscillating atomic force microscopy cantilevers in viscous fluids, Journal of Applied Physics, 97, 074907 (2005). doi:10.1063/1.1873060.

[25] J. Galvan-Miyoshi, J. Delgado, R. Castillo, Diffusing wave spectroscopy in Maxwellian fluids, The European Physical Journal E, 26, 369-377 (2008). doi:10.1140/epje/i2007-10335-8. 